\title{
Transmission electron microscopy study of CoMnO catalyst nanoparticles
}

Jinglong Guo ${ }^{1}$, Peng Wang ${ }^{2}$, Jordi Cabana ${ }^{3}$ and Robert Klie ${ }^{4}$

${ }^{1}$ University of Illinois at Chicago, Cupertino, California, United States, ${ }^{2}$ University of Illinois at Chicago, United States, ${ }^{3}$ Joint Center for Energy Storage Research, United States, ${ }^{4}$ University of Illinois at Chicago, Chicago, Illinois, United States

Development of high-efficiency and stable electrochemical materials is necessary for a sustainable and clean energy society.[1] High-performance functional electrocatalysts for oxygen evolution reactions (OER) play key roles in many energy conversion and storage technologies, such as rechargeable metal-air battery or for the production of hydrogen. [2]-[4]Traditionally, expensive noble-metal-based catalysts restrict the deployment and commercialization for sustainable energy devices. [5] Spinel oxides are excellent electric conductors and have good stability in alkaline solution under high overpotential, which makes it suitable for catalytical reaction. [5] The most commonly used spinel ORE catalysts are iron-based and cobalt-based spinel compounds. Cobalt-manganese based spinel oxide nanoparticles exhibit bifunctional properties as oxygen evolution/reduction reaction (OER/ORR) catalysts.[6], [7] In this work, we will utilize scanning transmission electron microscopy (STEM) imaging and electron energy loss spectrum (EELS) to examine the chemical composition and atomic structure of CoxMn3-xO4 nanoparticle made with ionic liquid. The core-shell CoxMn3-xO4 nanoparticles were synthesized by one-pot two-step procedure with additional ionic liquid. The electron microscopy characterization is acquired by JEOL-JEM ARM200CF equipped with Gatan continuum EEL spectrometer. The fine structure of oxygen $\mathrm{K}$ edge from nanoparticle shell and core will also be extracted and compared. Figure 1 shows high angle annular dark field (HAADF) STEM image with elemental atomic percentage maps of oxygen, manganese and cobalt. The atomic percentage line profile of $\mathrm{Co}, \mathrm{Mn}$ and $\mathrm{O}$ are extracted from the white arrow. The HAADF image shows the nanoparticles are in round shape and $12 \mathrm{~nm}$ in diameter. From the elemental mapping, it is clear to see that nanoparticle have core-shell structure. The outer shell has high concentration of cobalt. The cobalt-rich shell is about $4 \mathrm{~nm}$ thick and has highest cobalt concentration of $20 \%$ At. An atomic resolution HAADF image of CoxMn3-xO4 nanoparticles is shown in Figure 2, the FFTs of selected areas in core and shell are exhibited on the right side of Figure 2. The atomic HAADF shows spinel structure on [001] zone axis. There is significant structural difference between core and shell region. Based the FFT and atomic image, we found the deficiency of tetrahedral sites of spinel structure in the shell region. It is believed that the ionic liquid used during nanoparticles synthesis introduced such tetrahedral sites deficiency. The electrochemical performance of core-shell CoxMn3-xO4 nanoparticles will be corelated with the structural properties, chemical composition and EELS fine structure analysis. 



Figure 1. EELS elemental distribution mapping of $\mathrm{CoxMn3}-\mathrm{xO} 4$ with elemental atomic percentage line profile of $\mathrm{Co}, \mathrm{Mn}$ and $\mathrm{O}$ in CoxMn3-xO4-Vo nanoparticles.

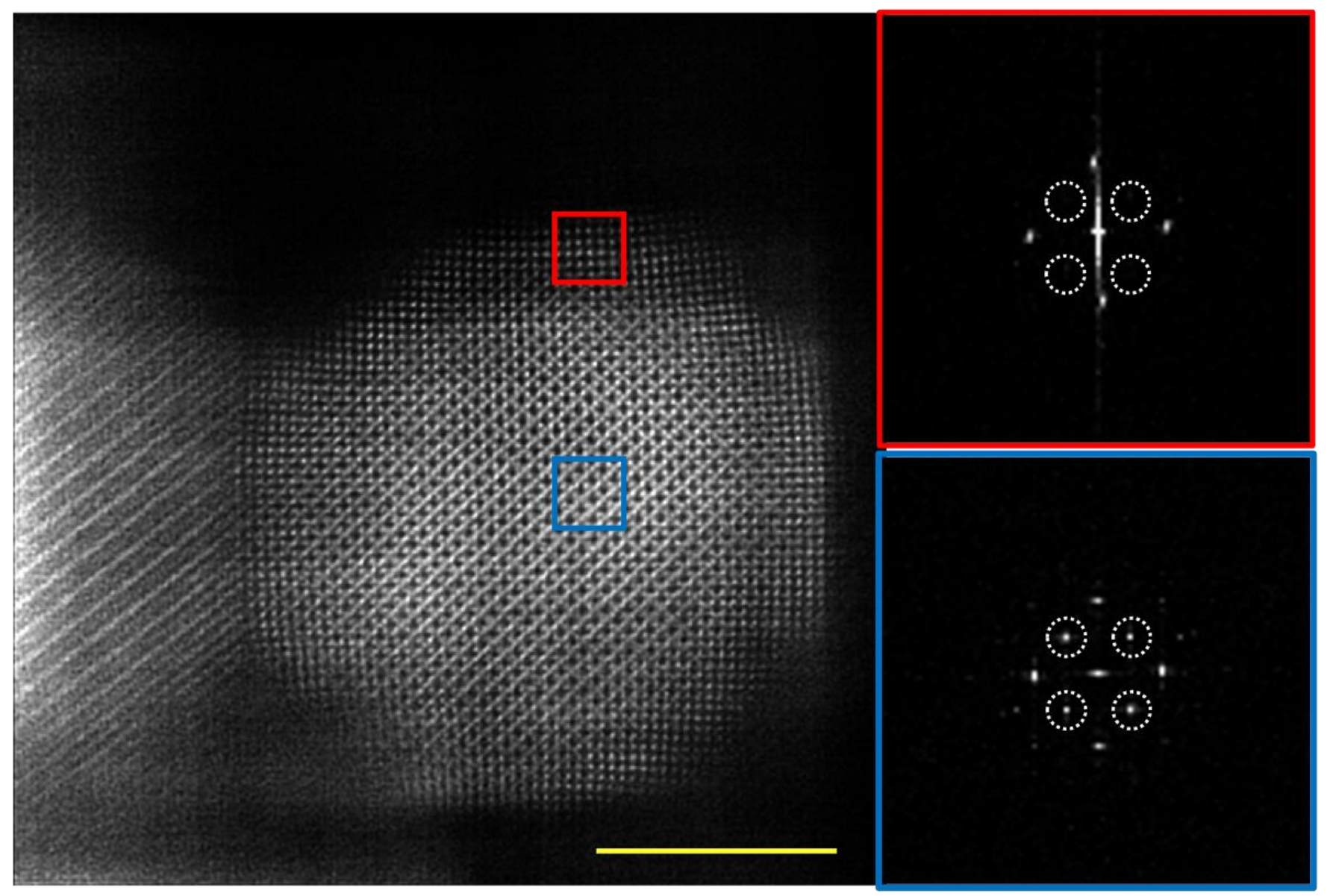


Figure 2. Atomic resolution HAADF image of CoxMn3-xO4 nanoparticles. The FFTs of core and shell regions is shown on the right from corresponding-colored regions.

\section{References}

[1] Z. W. She, J. Kibsgaard, C. F. Dickens, I. Chorkendorff, J. K. Nørskov, and T. F. Jaramillo, "Combining theory and experiment in electrocatalysis: Insights into materials design," Science (80-. )., vol. 355, no. 6321, 2017, doi: 10.1126/science.aad4998.

[2] P. G. Bruce, S. A. Freunberger, L. J. Hardwick, and J.-M. Tarascon, "Li-O2 and Li-S batteries with high energy storage," Nat. Mater., vol. 11, no. 1, pp. 19-29, 2012, doi: 10.1038/nmat3191.

[3] L. Malavasi, C. A. J. Fisher, and M. S. Islam, "Oxide-ion and proton conducting electrolyte materials for clean energy applications: structural and mechanistic features," Chem. Soc. Rev., vol. 39, no. 11, pp. 43704387, 2010.

[4] K. Kinoshita, Electrochemical oxygen technology, vol. 30. John Wiley \& Sons, 1992.

[5] N. T. Suen, S. F. Hung, Q. Quan, N. Zhang, Y. J. Xu, and H. M. Chen, "Electrocatalysis for the oxygen evolution reaction: Recent development and future perspectives," Chem. Soc. Rev., vol. 46, no. 2, pp. 337365, 2017, doi: 10.1039/c6cs00328a.

[6] C. Li, X. Han, F. Cheng, Y. Hu, C. Chen, and J. Chen, "Phase and composition controllable synthesis of cobalt manganese spinel nanoparticles towards efficient oxygen electrocatalysis," Nat. Commun., vol. 6, pp. 4-11, 2015, doi: 10.1038/ncomms8345.

[7] Z. Luo et al., "Mn3O4@CoMn2O4-CoxOy Nanoparticles: Partial Cation Exchange Synthesis and Electrocatalytic Properties toward the Oxygen Reduction and Evolution Reactions," ACS Appl. Mater. Interfaces, vol. 8, no. 27, pp. 17435-17444, 2016, doi: 10.1021/acsami.6b02786. 